\title{
Online Learning of Robust Facial Feature Trackers
}

\author{
Tim Sheerman-Chase, Eng-Jon Ong and Richard Bowden \\ CVSSP, University of Surrey, Guildford, Surrey GU2 7XH, United Kingdom \\ t. sheerman-chase, e.ong,r.bowden@surrey.ac.uk
}

\begin{abstract}
This paper presents a head pose and facial feature estimation technique that works over a wide range of pose variations without a priori knowledge of the appearance of the face. Using simple LK trackers, head pose is estimated by Levenberg-Marquardt (LM) pose estimation using the feature tracking as constraints. Factored sampling and RANSAC are employed to both provide a robust pose estimate and identify tracker drift by constraining outliers in the estimation process. The system provides both a head pose estimate and the position of facial features and is capable of tracking over a wide range of head poses.
\end{abstract}

\section{Introduction}

This paper presents an approach for the tracking of facial features and pose estimate of the head throughout a video sequence without an a priori model of appearance. The approach uses online learning to build a model of appearance on-the-fly using a generic 3D shape model to remove tracking drift inherent to online approaches.

For many applications involving the face, the accurate tracking of facial features is an important first step that precedes further processing e.g. identity verification, expression or action recognition. However, robust tracking of facial features is very challenging due to changing facial expression and pose. Additionally, one needs to be able to cope with self occlusions and lighting changes. For these reasons, attempting to robustly track facial features without a priori knowledge of their appearance is a difficult task. Appearance can be learn on-the-fly, however such approaches can fail drastically through the accumulation of errors during the learning process. While the appearance of the face may vary between individuals and settings, structurally they are the same and this structure can be used to reduce errors during the online learning process.

Existing work tends to address the above problems in two ways: 2D/3D model-based approaches; or posespecific piecewise tracking models. For model based approaches, a popular method is to use a $2 \mathrm{D}$ model for the face $[5,13,9,14]$, but these models only approximate the shape of the face at near frontal head pose. Alternatively, various $3 \mathrm{D}$ head models can be used [7, 1, 12, 4, 2]. These more complex models require accurate initialization and are computationally expensive. 3D head models can range from simple cylindrical and ellipsoidal models to complex polygonal approximation, usually used for pose estimation. Many of these techniques use template update, by incrementally modifying the expected appearance to correspond with the observed appearance and to achieve pose invariant tracking. More accurate model-based facial feature tracking can be achieved using a realistic 3D face model of sufficient detail for rendering. This allows for a realistic reconstruction of the visual appearance of the face [11]. However, these models are complicated and deforming the model to fit the image is usually non-trivial.

The next class of approaches couples a set of posespecific trackers with a switching mechanism (e.g. pose estimator) to decide which of these trackers to use. An example is proposed by Kanaujia et al.2006 [6] where multiple 2D pose-specific Active Shape Models (ASMs) are coupled with a switching mechanism using SIFT descriptors. Peyras et al.2008 [10] used a pool of Active Appearance Models (AAMs) that were specialized at different poses and expressions but not robust to changes in illumination. However, in order to train a reliable ASM or AAM model, a large amount of training data in the form of labeled shapes was needed.

This paper proposes a novel framework (Figure 1) that combines elements of both approaches described above. Specifically, sets of pose-specific facial feature trackers are integrated into a robust pose estimation system detailed in Section 2.5. The robust estimation of pose based on noisy tracking data is a novel extension of Random Sample Consensus (RANSAC) [3]. The tracking is initialised using alignment of a generic 3D model based on features that are easily identified using feature localisation. Following a detailed description of our approach in section 2, results presented in section 3, demonstrate the accuracy of the approach. Finally, conclusions are drawn in section 4. 


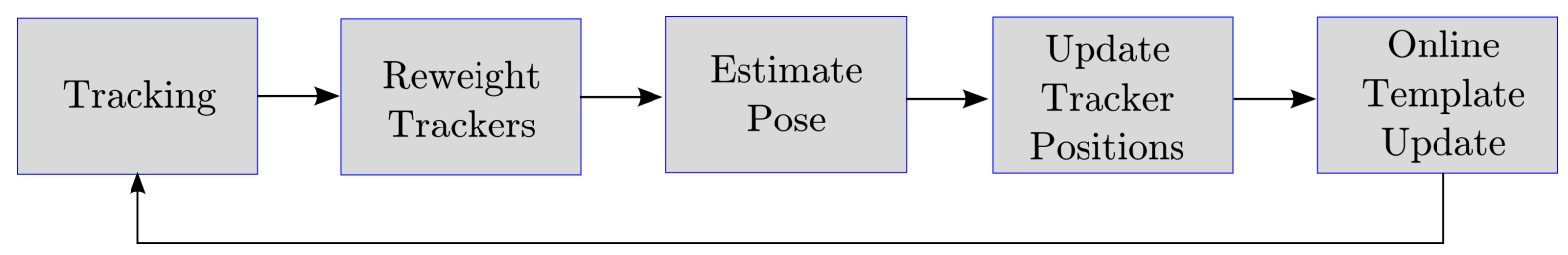

Figure 1. Overview of online tracker system.

\section{Online Facial Feature Tracking}

An overview of the tracking processing is given in Figure 1. Online tracking of the face is performed by initialisation on the first frame of the sequence based on a set of easily identifiable landmarks around the mouth, nose and eyes. From these initial position estimates, the pose of the head is determined using a generic face mesh and LM minimisation. A larger set of features are then back projected into the image to initialise a set of trackers over the face region. The 3D face mesh was obtained using a commercial 3dMD system.

Template trackers are initialised at each feature position. The template is a square image patch centred on the feature and Lucas Kanade tracking is used to perform template alignment.

The first iteration of the sequence is described in section 2.1 in which each feature is represented by a single template. This removes the requirement for weights to be considered during the first iteration. However, as tracking progresses through the sequence, the new appearance of the tracked features are stored for later use. Later iterations have multiple templates per facial feature point with calculated confidence weights (described in section 2.4). The set of templates represent the appearance of a feature at various poses and the confidence weights give an indication of which templates match the current pose of the head.

As the feature may revert to a previously observed appearance, all retained templates are used to track at each frame. This is feasible as the templates are small, finite in number and relatively cheap to compute in an LK framework. The confidence weighting, assigned to each template tracker, is used with RANSAC and factored sampling to determine a robust head pose at each iteration. Weighting is determined by measuring which trackers are in good agreement with the back projected head model and following pose estimation are used to control the degree of correction to feature positions. The feature positions are corrected/updated based on the weighted average of the tracker position and the back projected model. Once the tracker positions have been constrained by the model pose estimate, the templates are updated with new appearances from the current frame. The process then reiterates by repeating this procedure on each consecutive frame of video.

The approach enables tracking and adaptation to previ-

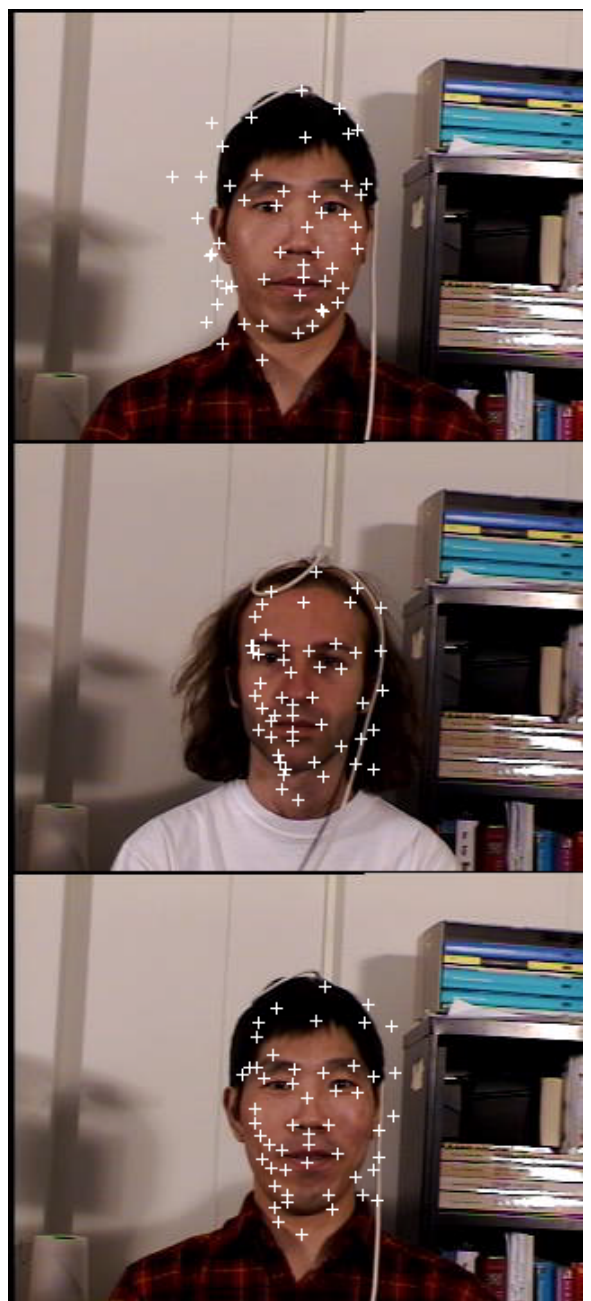

Figure 2. Examples of poor initial head pose alignment. The tracker points on the background indicate the face mesh is not in agreement at the start of the sequence.

ously unseen pose angles in the video sequence. However, the constraints applied by the 3D head model prevent tracking drift during online learning. We will now discuss each of these processes in turn.

\subsection{Tracking Initialisation}

The face appearance is represented by a set of $J$ features, each of which is a tuple $\left(\mathbf{M}_{j}, \mathbf{p}_{j}, \mathbf{z}_{j}\right)$ where $1<j<J, \mathbf{M}_{j}$ is a set of $\mathbf{n}_{j}$ templates, $\mathbf{p}_{j} \in \mathbb{R}^{3}$ is a face mesh position and 
$\mathbf{z}_{j} \in \mathbb{R}^{2}$ is an image position. The set of templates $\mathbf{M}_{j}$ is a tuple $\left(\mathbf{K}_{i, j}, \mathbf{w}_{i, j}, \mathbf{a}_{i, j}\right)$ comprising of $\mathbf{n}$ image patches $\mathbf{K}_{i, j}$, a weight $\mathbf{w}_{i, j}$, and an image position $\mathbf{a}_{i, j}$ where $1<i<\mathbf{n}$ $\left(\mathbf{K}_{i} \in \mathbb{R}^{\mathbf{n}_{j}}, \mathbf{w}_{i} \in \mathbb{R}^{\mathbf{n}_{j}}, \mathbf{a}_{i} \in \mathbb{R}^{2 \times \mathbf{n}_{j}}\right)$.

At initialisation, 19 key points on the mesh are identified corresponding to simple features that are easy to identify using detection, such as the corners of the mouth, eyes, nose and eyebrows as depicted in Figure 3. From these known points, the $3 \mathrm{D}$ pose of the head is estimated using equation (1) and a further 32 evenly positioned features over the facial model are backprojected into the image. For each of the 51 feature points $(J=51)$, the initial template within the image, $\mathbf{K}_{i, j}$, is retained at each of the feature positions $\mathbf{a}_{i}^{F=0}$ along with the estimated rotational pose of the head $\mathbf{P}_{i, j}\left(\mathbf{P}_{i, j} \in \mathbb{R}^{3 \times \mathbf{n}_{j}}\right)$. As each feature initially corresponds to only one template $\left(\mathbf{n}_{j}=1\right)$, initial weights are set to be equal $\left(\mathbf{w}_{i, j}=1\right)$.

Lucas Kanade tracking is then used to estimate the positions of all features on the second frame.

\subsection{Head Pose Estimation by LM}

Many existing pose estimation techniques use feature tracking/detection and iterative model fitting. LM minimization can be used to determine pose from a point cloud when point correspondence is known [8]. The pose is estimated by minimizing the cost function:

$$
\mathcal{F}(\mathbf{R}, \mathbf{t})=\sum_{j=1}^{J} \sum_{i=1}^{\mathbf{n}_{j}}\left\|\mathbf{a}_{i, j}-\operatorname{proj}\left(\mathbf{R p}_{i}+\mathbf{t}\right)\right\|^{2}
$$

where $\operatorname{proj}()$ is the projection function, $\mathbf{R}$ is the current rotation matrix and $\mathbf{t}$ is the head translation. The resulting $\mathbf{R}$ and $\mathbf{t}$ matrices represent the estimated head pose (where $\mathbf{R}$ is the rotation matrix corresponding to the Euler angles $\left\{R_{\text {pitch }}, R_{\text {roll }}, R_{\text {yaw }}\right\}$ and $\mathbf{t}$ is the head translation $\left.\left\{t_{x}, t_{y}, t_{z}\right\}\right)$. Perspective geometry is used for the projection function.

\subsection{Online Learning}

As the appearance of facial features is dependent on the direction of view, periodically retraining a tracker can help the tracker adapt to new appearances. However, care must be taken that online tracker updates are not used excessively as this causes tracking drift. Drift can be minimized by performing a template update only when necessary to adapt to new appearances. The central idea of this system is to balance adaptation to the changing appearance of a feature without introducing tracker drift.

Each feature has one or more tracker templates $\mathbf{K}_{i, j}$ with a corresponding head pose estimate $\mathbf{P}_{i, j}$ at which the template was created. At each iteration, the current head pose estimate $R$ is compared to the pose at which templates were added to the model. If no template exists within an angle $\mathbf{c}_{i}$ of the current frames estimated head pose, an additional template is added to the model for that feature. To enable gradual adaptation to new appearance, each feature has a independent threshold to prevent collection of new templates from all features simultaneously. All previous templates are retained. Consequently, as the head moves in the image, the model gradually accumulates a set of templates representative of the appearance of features at all head orientations.

\subsection{Tracking with Multiple Templates}

Tracking proceeds in a similar manner to the first iteration for all subsequent frames, except each landmark on the face has multiple templates that may represent its appearance. Lucas Kanade tracking is used to estimate each template position $\mathbf{a}_{i, j}^{F}$ on frame $F$ with the initial tracker position taken from the corresponding landmark $\mathbf{z}_{i}^{F-1}$ on frame $F-1$.

For a specific landmark, some templates will be more suited for tracking than others due to it having various possible appearances. To prioritise these trackers, a weighting of each template $\mathbf{w}_{i, j}$ is calculated based on their tracking agreement with the face model on frame $F-1$. This enables trackers with good performance to be preferred over trackers with poor performance in the pose estimation step. The weightings are assigned as follows:

$$
\mathbf{w}_{i, j}=e^{-\frac{\mathbf{u}_{i, j}}{s}}
$$

where $\mathbf{u}_{i, j}$ is the difference between the tracker prediction and the back projected mesh node $\mathbf{p}_{i}$ in pixels $\left(\mathbf{u}_{i} \in\right.$ $\mathbb{R}^{\mathbf{n}_{i}}$ ) and $s$ is the agreement scaling factor.

\subsection{Estimate pose using Weighted RANSAC LM}

Although the use of a confidence weighting reduces the effect of poorly performing trackers on the pose estimate, occasionally a tracker of higher confidence undergoes drift. Conventional LM pose estimation is not robust to outliers resulting from tracker drift. A robust framework that incorporates tracker confidence weightings is described. This framework is an extension to the Random Sampling and Consensus (RANSAC) method to incorporate factored sampling. To enable the preference of high confidence points over points with lower confidence, equation (1) can be modified to incorporate weighting term:

$$
\mathcal{F}(\mathbf{R}, \mathbf{t})=\sum_{i=1}^{J} \sum_{j=1}^{\mathbf{n}_{i}} \mathbf{w}_{i, j} \cdot\left\|\mathbf{a}_{i, j}-\operatorname{proj}\left(\mathbf{R p}_{i}+\mathbf{t}\right)\right\|^{2}
$$

For a single RANSAC iteration, a random subset $\rho$ of $l$ 


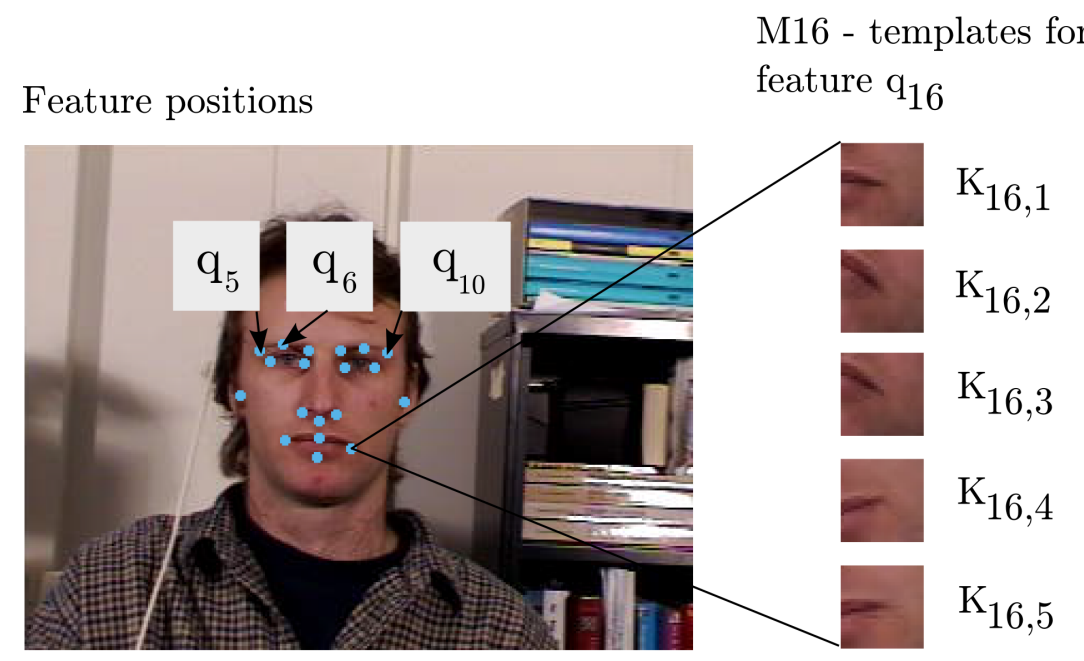

Figure 3. Position of features used to initialize and track the face. Multiple templates $M_{i}$ at various poses represent the appearance of each feature.

points is sampled from the distribution $p\left(\mu_{i, j}\right)$ :

$$
p\left(\mu_{i, j}\right)=\frac{\mathbf{w}_{i, j}}{\sum \mathbf{w}}
$$

Following this, an LM pose model fit is performed on random subset $\rho$. The point confidence weights $\mathbf{w}$ are used in this minimization. Points not in set $\rho$ are checked to determine if they are in agreement with model. Support is calculated by summing the agreement with the model where the agreement is determined by the distance $\| \mathbf{a}_{i, j}-$ $\operatorname{proj}\left(\mathbf{R} \mathbf{p}_{i}+\mathbf{t}\right) \|$. If the proportion of the weight of inliers, compared to the weight of all points $\sum_{i=1}^{J} \sum_{j=1}^{\mathbf{n}_{i}} \mathbf{w}_{i, j}$, is less than a good agreement threshold $\beta$, this iteration model is discarded. The model fit error $E$ is calculated are follows:

$$
E=\sum_{i=1}^{J} \sum_{j=1}^{\mathbf{n}_{i}}\left\|\mathbf{a}_{i, j}-\operatorname{proj}\left(\mathbf{p}_{i}\right)\right\| \cdot \mathbf{w}_{i, j}
$$

where $E$ is the model fit in pixels.

If the model fit error is lower than any previous model fit, it is stored as the new best model fit. We overcome the problem of parameter selection of $\beta$ by iterating the process over decreasing $\beta$ values until a RANSAC solution is found.

\subsection{Update tracker positions}

As described above, each landmark has multiple templates that represent its appearance. To determine a final landmark position on frame $F$ and correct any trackers that drift, the highest weighted template position is combined with the corresponding 3D mesh point back projected onto the image. As LK is an iterative tracking scheme, under normal conditions, tracking drift can result in total failure once the tracker is outside the basin of convergence of the LK optimisation. By correcting trackers, this enables points with a low confidence to be corrected to within the tracker's basin of convergence.

Tracking performance for features that are occluded or have undergone drift are naturally expected to be inaccurate and will therefore have a low weight. A low weight will result in playing little or no part in the RANSAC estimation and they will therefore be corrected using the position from the back projected mesh at this stage of the algorithm.

$$
\begin{aligned}
\mathbf{z}_{i} & =\mathbf{w}_{i, j} \cdot \mathbf{a}_{i, j}+\left(1-\mathbf{w}_{i, j}\right) \cdot \operatorname{proj}\left(\mathbf{p}_{i}\right) \mid j \\
& =\arg \max _{j} \mathbf{w}_{i, j}
\end{aligned}
$$

where $\mathbf{z}_{i}$ is the new tracker position, $\mathbf{a}_{i, j}$ is the highest weighted prediction $\mathbf{w}_{i, j}$.

Processing of this frame concludes by the addition of templates to the model as described in section 2.3.

\section{Experimental Results}

The proposed system was tested in two experiments. The first determined the head pose estimation accuracy and the second measured the feature tracking accuracy. All tests were performed on a "uniform lighting" video sequences provided by Boston University [7]. The data set contains 45 video sequences, containing 5 subjects with a resolution of $320 \times 240$ pixels, which all begin with a frontal view of the face. The data includes head pose ground truth recorded using an electromagnetic sensor with an angular accuracy of 0.5 degrees in ideal conditions. 


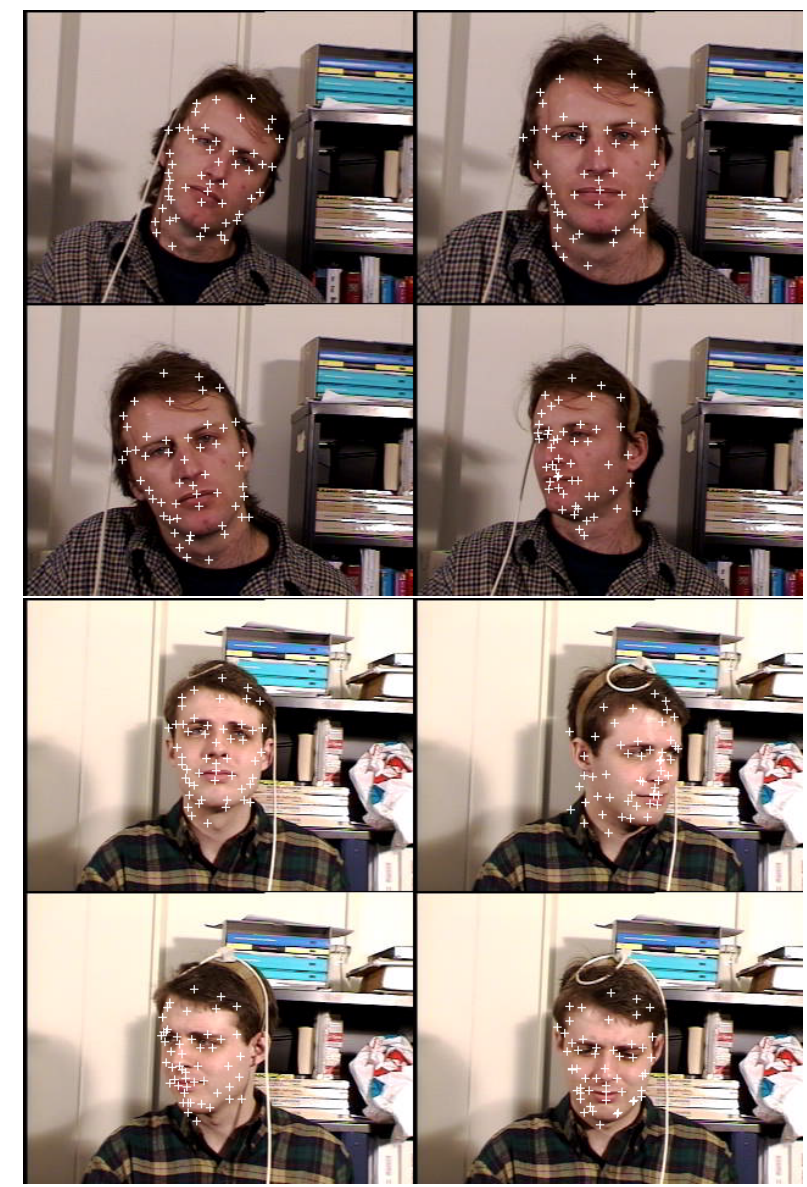

Figure 4. Examples showing online tracking of feature positions at various pose angles of subjects JAM and JIM. Accurate tracking is possible from various view angles of the face.

\subsection{Head Pose Estimation}

For robust pose estimation, the RANSAC was set to use a minimum number of model points $l=6$. The LK template size was $25 \times 25$ pixels, the agreement scaling factor $s=$ 10.0 and the retraining thresholds was randomly selected in the range $\mathbf{c}_{i}=2 \pm 1$.

Testing the online LK system on 45 sequences, the average estimation error for pitch, roll and yaw were $3.9^{\circ}, 3.1^{\circ}$, $4.2^{\circ}$ respectively. This is compared to the performance of other methods in Table 1. A comparison of estimated pose with ground truth for two sequences is shown in Figure 5. These graphs show the ground truth pose for Roll, Pitch and Yaw as a dashed (red) line and the predicted pose as solid (blue). As can be seen, the prediction largely follows the ground truth, although there is a noticeable time lag between the signals which we suspect is due to smoothing on the ground truth as it is the ground truth which lags behind the prediction. The estimated pose also has a tendency to underpredict at extremes of pose. The jitter in the prediction is a direct result of the RANSAC LM estimation, as no

\begin{tabular}{|c|c|c|c|}
\hline & \multicolumn{3}{|c|}{ Average Error (Deg) } \\
Method & Pitch & Roll & Yaw \\
\hline Proposed method & 3.9 & 3.1 & 4.2 \\
Jang and Kanade 2008[4] & 3.7 & 4.6 & 2.1 \\
Choi and Kim 2008[2], Cylinder & 4.4 & 5.2 & 2.5 \\
Choi and Kim 2008[2], Ellipsoid & 3.9 & 4.0 & 2.8 \\
\hline
\end{tabular}

Table 1. Comparison of performance with other head pose estimation methods.

\begin{tabular}{|c|c|c|c|c|c|}
\hline \multicolumn{6}{|c|}{ Average Error (pixels) for Subject } \\
JAM & JIM & LLM & SSM & VAM & Overall \\
\hline 3.1 & 3.8 & 5.0 & 3.2 & 3.5 & 3.7 \\
\hline
\end{tabular}

Table 2. Results of 2D facial feature tracking 45 sequences.

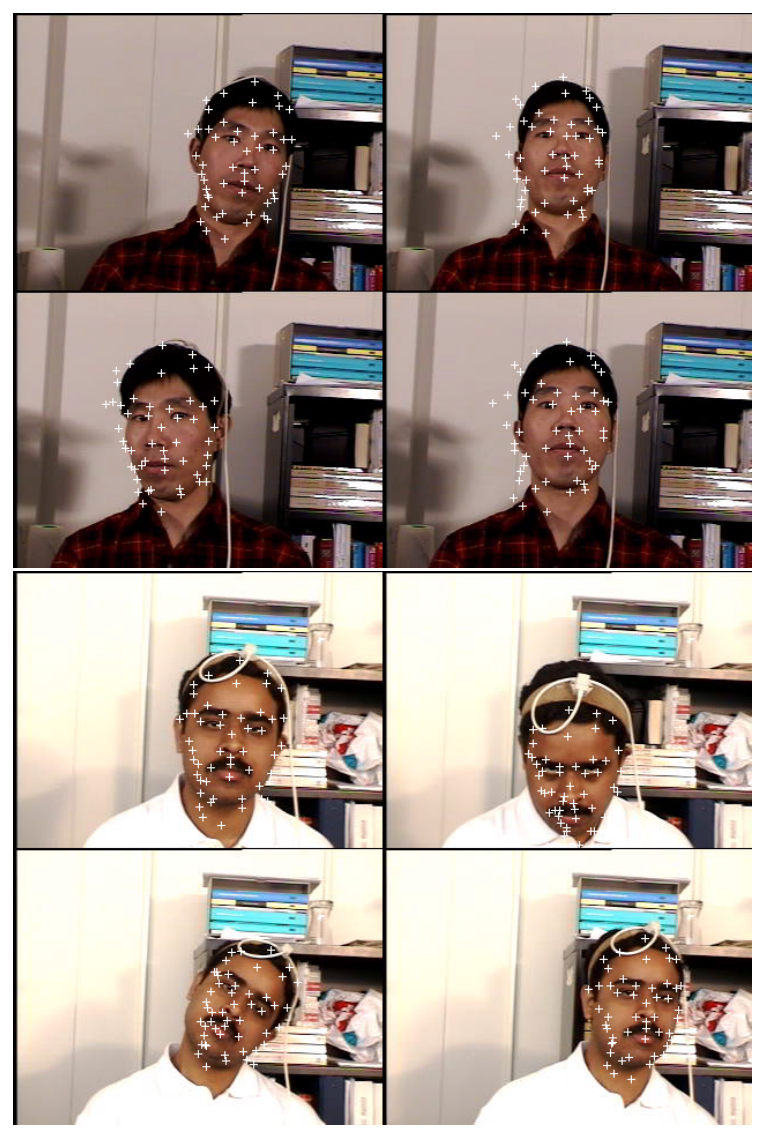

Figure 6. Examples showing online tracking of feature positions at various pose angles of subjects LLM and SSM.

temporal constraints or smoothing were applied.

The system initialized accurately on most individuals but was consistently inaccurate on one subject due to the face shape being noticeably different to the generic face mesh (see figure 2).

\subsection{Facial Feature Tracking}

In addition to the pose estimate, the feature point accuracy is also considered as the tracking of individual fea- 

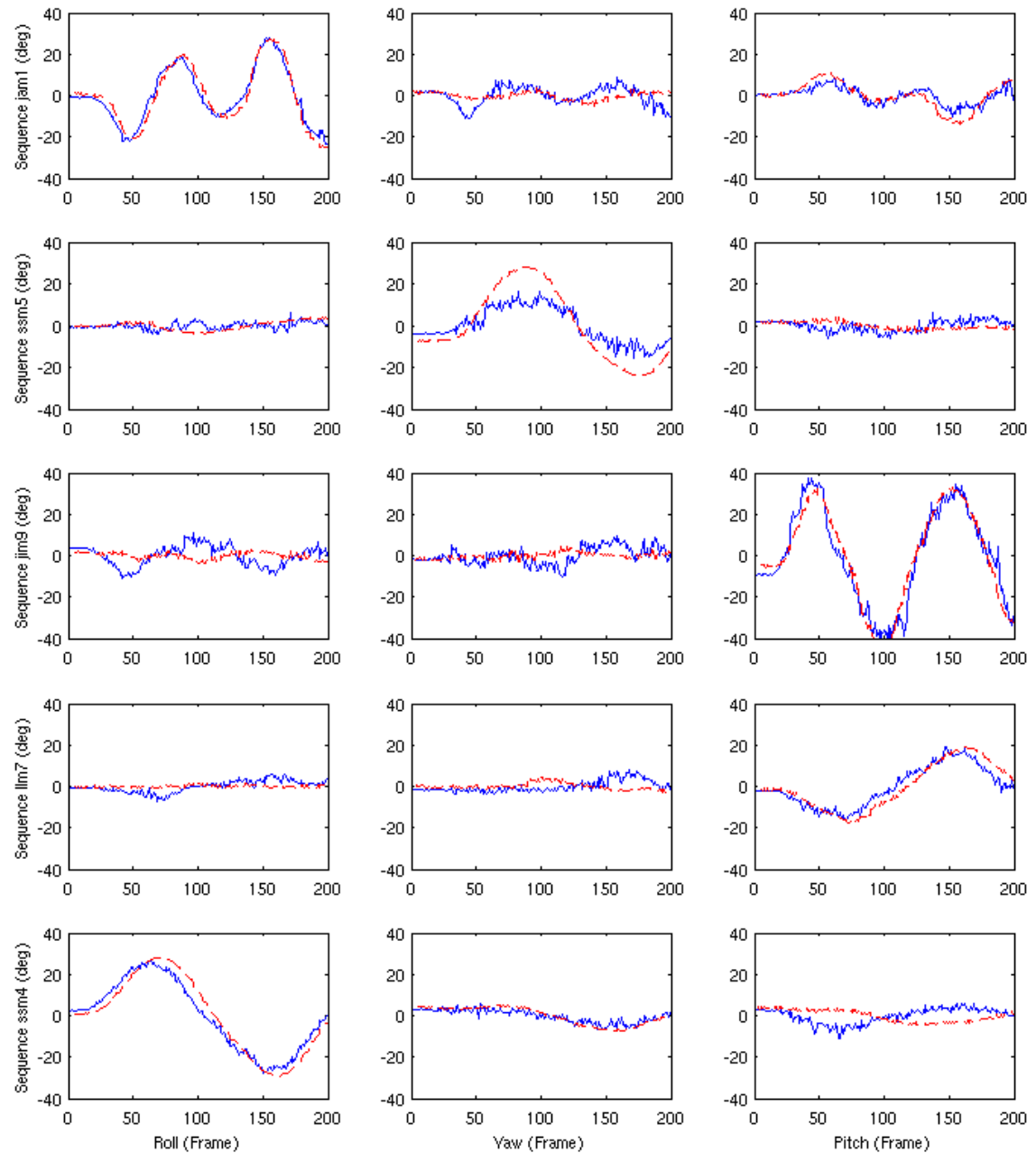

Figure 5. Examples of head pose estimation for five sequences. The variation for three rotation axis are shown for each sequence. Blue solid line is predicted pose. Red dashed line is ground truth.

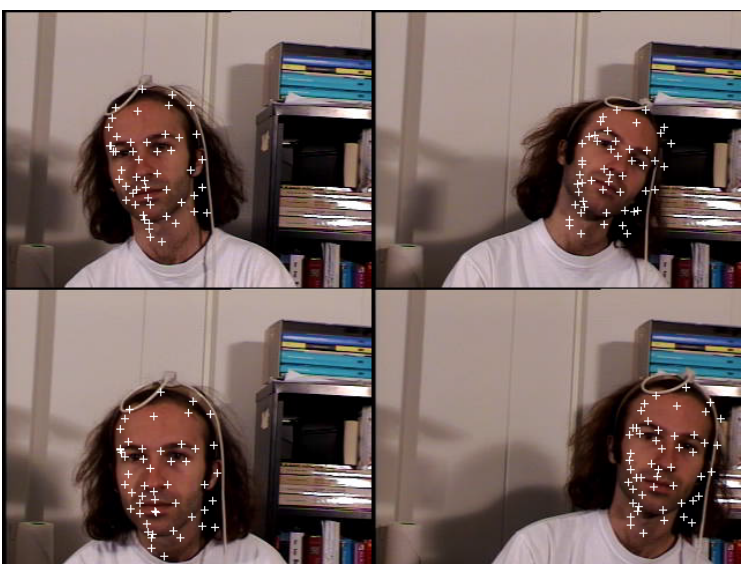

Figure 7. Examples showing online tracking of feature positions at various pose angles of subjects VAM. ture points and their motion is important for other applications such as expression recognition. The tracking performance was measured by comparing predicted feature positions $\mathbf{z}_{j}$ to the ground truth feature position. The test sequences again used the 45 sequences in the "uniform lighting" Boston University data set. Only the 17 features on the face were included in the performance assessment (see fig 3 ); the ear positions could not be reliably tracked due to frequent occlusions. Table 2 shows the average error in pixels for 5 subjects from the database (labelled JAM, JIM, LLM, SSM and VAM). For all 45 sequences the average error was 3.7 pixels. Ground truth positions were semi manually annotated for the sequences. To find the noise level in this ground truth, two sequences were fully manually ground truthed and compared to the semi-automatic method. The average position error was 2.7 pixels.

7.

Examples of online tracking are shown in Figure 4, 6 and 


\section{Conclusions}

This paper described a system that automatically estimates head pose and facial feature positions without a priori knowledge of the appearance of the face. The system uses robust LM pose estimation within a RANSAC framework to constrain features during on-line learning. The constraints are weighted based on the tracking agreement with a 3D face mesh. The online learning rate is controlled by changes in head pose. Accurate estimation of head pose is achieved over a wide range of poses resulting in comparable performance to other state-of-the-art approaches.

\section{Future Work}

For some subjects, the initial head pose estimate is poor due to differences in head shape as the system uses a rigid mean model. This also has implications to radical changes in expression which are not represented in the model. Future work will address robustness to expression and variation in head shape and the inclusion of a variable shape model could overcome these issues.

\section{Acknowledgements}

This work has been largely supported by the EPSRC project LILiR and in part by the FP7 project DIPLECS.

\section{References}

[1] G. Aggarwal, A. Veeraraghavan, and R. Chellappa. 3D facial pose tracking in uncalibrated videos. In Proc. of Intl. Conf. on Pattern Recognition and Machine Intelligence, volume 3776, pages 515-520, 2005.

[2] S. Choi and D. Kim. Robust head tracking using 3D ellipsoidal head model in particle filter. Pattern Recogn., 41(9):2901-2915, 2008.

[3] M. A. Fischler and R. C. Bolles. Random sample consensus: a paradigm for model fitting with applications to image analysis and automated cartography. Commun. ACM, 24(6):381395, 1981.

[4] J.-S. Jang and T. Kanade. Robust 3D head tracking by online feature registration. In 8th IEEE Int'l Conference on Automatic Face and Gesture Recognition, 2008.

[5] A. D. Jepson, D. J. Fleet, and T. F. El-Maraghi. Robust online appearance models for visual tracking. Pattern Analysis and Machine Intelligence, IEEE Transactions on, 25(10):12961311, 2003.

[6] A. Kanaujia, Y. Huang, and D. Metaxas. Emblem detections by tracking facial features. In CVPRW '06: Proceedings of the 2006 Conference on Computer Vision and Pattern Recognition Workshop, page 108, Washington, DC, USA, 2006. IEEE Computer Society.

[7] M. La Cascia and S. Sclaroff. Fast, reliable head tracking under varying illumination: An approach based on robust registration of texture-mapped 3D models. In IEEE Transactions on Pattern Analysis and Machine Intelligence, 22:322-336, 2000.

[8] Z. Liu and Z. Zhang. Robust head motion computation by taking advantage of physical properties. In Workshop on $\mathrm{Hu}$ man Motion, pages 73-77, 2000.

[9] K. Okuma, A. Taleghani, O. D. Freitas, J. J. Little, and D. G. Lowe. A boosted particle filter: Multitarget detection and tracking. In Proceedings of European Conference on Computer Vision, pages 28-39, 2004.

[10] J. Peyras, A. Bartoli, and S. Khoualed. Pools of AAMs: Towards automatically fitting any face image. In Proceedings of the Nineth British Machine Vision Conference, Leeds, UK, September 2008.

[11] D. Terzopoulos and K. Waters. Analysis and synthesis of facial image sequences using physical and anatomical models. IEEE PAMI, 15(6), June 1993.

[12] J. Xiao, T. Moriyama, T. Kanade, and J. Cohn. Robust full-motion recovery of head by dynamic templates and reregistration techniques. International Journal of Imaging Systems and Technology, 13:85-94, September 2003.

[13] S. K. Zhou, R. Chellappa, and B. Moghaddam. Visual tracking and recognition using appearance-adaptive models in particle filters. IEEE Transactions on Image Processing, 13(11):1491-1506, 2004.

[14] Z. Zhu and Q. Ji. Real time 3d face pose tracking from an uncalibrated camera. In $C V P R W$, page 73, 2004. 